\title{
An upper limit prediction of the peak sunspot number for solar cycle 24
}

\author{
T. Podladchikova ${ }^{1, *}$, and R. Van der Linden ${ }^{2}$ \\ 1 Institute for Applied System Analysis, NTUU KPI, Ukraine, Solar-Terrestrial Center of Excellence, ROB, Belgium \\ *correspondng author: e-mail: tatyana@oma.be; tpodlad@gmail.com \\ 2 Solar-Terrestrial Center of Excellence, ROB, Belgium \\ e-mail: Ronald.VanderLinden@oma.be
}

Received 10 February 2011 / Accepted 02 May 2011

\section{ABSTRACT}

\begin{abstract}
In this paper we address the prediction of the maximum sunspot number in solar cycle 24 . We correlate the integral sunspot activity related to the variability of the cycle decrease speed in the declining phase with the height of the next sunspot maximum. Using this method we predict that the sunspot maximum of cycle 24 will be weaker than the previous one and its peak value will not exceed 72 .
\end{abstract}

Key words. 7536: solar activity cycle - 3238: prediction

\section{Introduction}

Forecasting of the peak of the sunspot cycle is highly important for space weather applications. At the present time, precursor methods are the most favored for the prediction of the strength of the next solar cycle (Kane 2008; Hathaway 2009). These precursor techniques often relate to geomagnetic activity levels near, or before, the time of solar cycle minimum (Sargent 1978; Ohl \& Ohl 1979; Feynman 1982; Gonzalez \& Schatten 1987; Thomson 1993; Wilson et al. 1998).

Predicting the amplitude of a solar cycle can be done using polar fields from the previous cycle as "precursors" of the next cycle (Schatten \& Sofia 1987).

The other class of precursor techniques that do not need a priori a physical understanding of the causal relations (i.e., that do not require any knowledge of the physics involved) is based on finding particular sunspot number characteristics that serve as indicators of the size of the next cycle (Ramaswamy 1977; Lantos 2006; Cameron \& Schüssler 2008; Brajša et al. 2009).

The physical explanation for how precursor methods work was suggested by Schatten et al. (1978), who used the reversed polar field built up after the solar maximum as a precursor indicator to the next solar cycle strength. Svalgaard et al. (2005) have reported about correlation between polar fields and sunspot activity of the next cycle.

In the framework of a dynamo model of the BabcockLeighton type, Cameron \& Schüssler (2007) have discussed the importance of the sunspot activity level during the declining phase and considered as a predictor the sunspot activity three years before the minimum. In our recent paper (Podladchikova et al. 2008) we extended the previous work of precursor methods based upon correlations between sunspot activity in the declining phase of a cycle and the strength of the next cycle by showing that the detailed variability of the sunspot number in the declining phase of the solar cycle is closely related to the rise and maximum of the following cycle. The method we use for the prediction is based on the estimation of the rate of decrease of the sunspot number in the declining phase. It allows predicting the solar cycle strength once the minimum of the previous cycle has passed. In the present analysis we applied this technique for the forecasting of the peak value for solar cycle 24 taking into account the present minimum of the solar activity.

\section{The relevant indicators for prediction}

Our technique was developed for the prediction of the maximum amplitude $R M_{k+1}$ of the 11-year solar cycle $k$ as described by the 13-month averaged sunspot number. For the analysis and estimation of the future solar activity we introduced an "integral activity" that is considered as a precursor of the next solar cycle. The integral activity of the sunspot cycle $S_{k},(k=1, \ldots, 23)$ of each cycle $k$ was determined as the area delimited by the cycle curves, as shown in Figure 1a (blue color). The integral activity of the declining phase $S_{k}^{\Delta}$ was defined by the area of the figure colored in gray (Fig. 1b). The area colored in red (Fig. 1a) is determined as $S_{k}^{\nabla}$.

To estimate the declining speed we compared the real sunspot number fall with the case of "uniform decrease" (the straight line $B_{k} A_{k+1}$ from the maximum to the minimum in Fig. 1b). Let us define $S_{k}^{+}$as the sum of the blue-colored areas lying above the straight line $B_{k} A_{k+1}$ for every $k$ (Fig. 1b). The area $S_{k}^{-}$represents the sum of the red-colored areas lying below the straight line $B_{k} A_{k+1}$ for every $k$ (Fig. 1b). The area $S_{k}^{+}$represents the "excess" activity and the area $S_{k}^{-}$characterizes the activity "shortfall" in comparison with a uniform decline.

The first created relevant indicator $\Delta_{k}=\frac{S_{k}^{+}-S_{k}^{-}}{R M_{k}}$ determines if the amplitude of the next cycle $R M_{k+1}$ will be larger or smaller compared to the current one. Nevertheless, the areas $S_{k}^{+}$and $S_{k}^{-}$are too small and the difference between these quantities is very sensitive to the errors of the smoothing. This is the principal disadvantage of this indicator. It motivated the construction of the second indicator that uses larger quantities that are less sensitive to the errors of the smoothing procedure.

The second relevant indicator $\frac{S_{k}}{S_{k}^{\nabla}}$ is used to get quantitative predictions about the increase or decrease of the ratio $\frac{R M_{k+1}}{R M_{k}}$. 


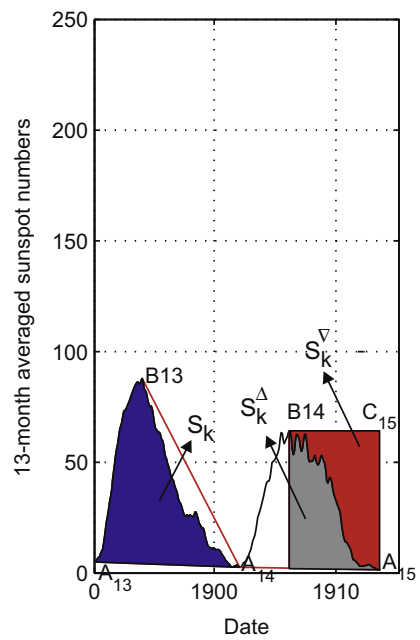

(a)

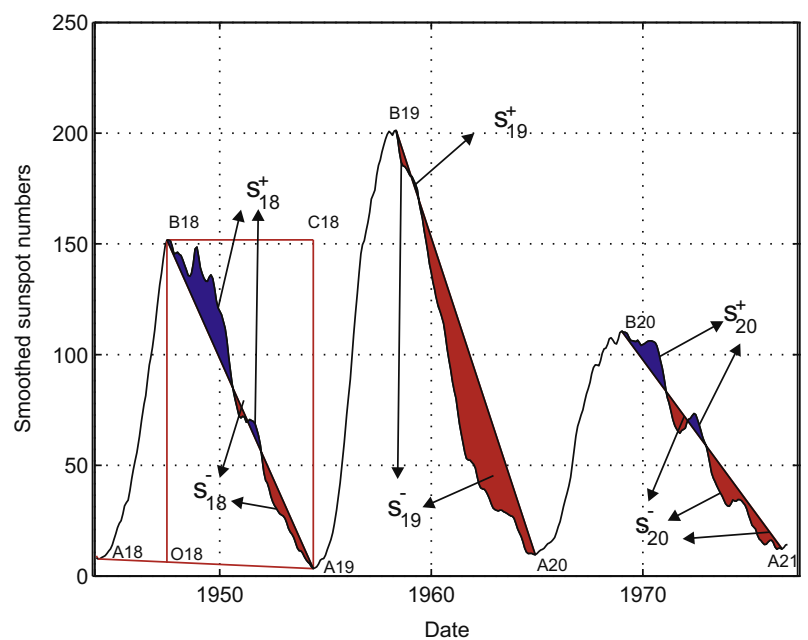

(b)

Fig. 1. (a) Evolution of the sunspot numbers during two 11 -year cycles $(k=13,14)$. (b) Variations of the solar activity during the declining

\section{The results of the peak sunspot number prediction}

The values of the first indicator $\Delta_{k}$ were compared with the values of $R M_{k+1}$. Figure 2 shows the maximum amplitude $R M_{k+1}$ and normalized value of the indicator $\delta_{k}=5.6 \Delta_{k}$.

As shown from Figure 2 the increase (decrease) of $\delta_{k}$ precedes the increase (decrease) of the maximum $R M_{k+1}$ for all the cycles $(k=1,3, \ldots, 22)$. The decrease of $\delta_{23}$ compared with $\delta_{22}$ predicts that cycle 24 will be weaker than cycle 23 .

The second indicator $\frac{S_{k}}{S_{k}^{V}}$ depends not only on the variability of the declining phase, but also on the cycle asymmetry. The sunspot cycles are typically asymmetric in shape with a rapid rise to maximum and a slower decline to minimum. The exceptions to this are some of the early cycles when the observations are sparse and more uncertain (Hathaway et al. 1999).

To obtain a reasonably accurate quantitative prediction for cycle 24 we examine the second relevant indicator $\frac{S_{k}}{S_{k}^{V}}$ only during the modern era (cycles 10-23).

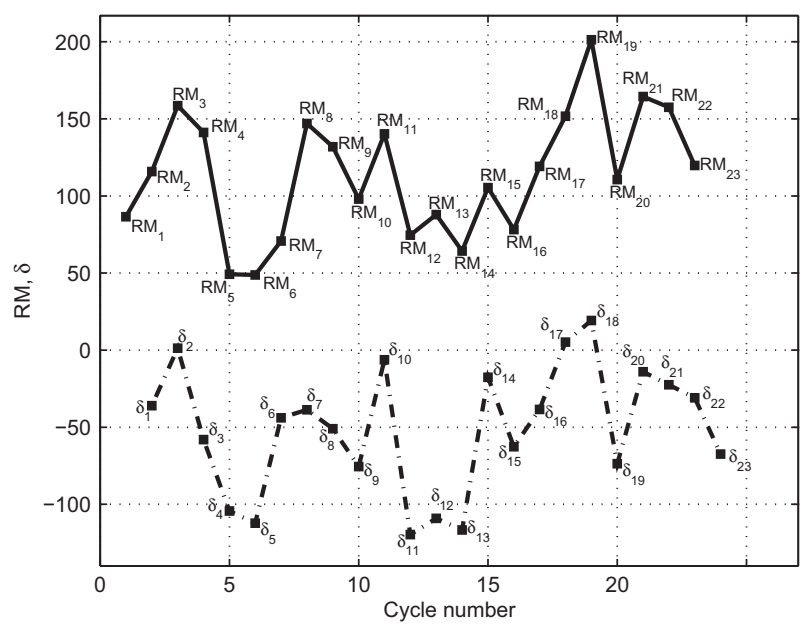

Fig. 2. The maxima $R M_{k}$ (solid line) compared to $\delta_{k-1}$ (dashed line) for cycles $k=1-23$. An increase (decrease) of $\delta_{k-1}$ precedes an increase (decrease) of the maxima of the solar cycle for all $k$. The decrease of $\delta_{23}$ compared with $\delta_{22}$ predicts that cycle 24 will be
Figure 3 shows the indicator $\frac{S_{k}}{S_{k}}(k=10,11, \ldots, 23)$ and the ratio of maximums $\frac{R M_{k+1}}{R M_{k}}(k=10,11, \ldots, 22)$.

As Figure 3 shows, the dynamics of indicator $\frac{S_{k}}{S_{k}^{V}}$ substantially follows that of the ratio $\frac{R M_{k+1}}{R M_{k}}$. A significant increase (decrease) of $\frac{S_{k}}{S_{k}^{V}}$ precedes a significant increase (decrease) of $\frac{R M_{k+1}}{R M_{k}}$. But for all $k=(11 \ldots 23)$ the distance between the ratios of maxima $\frac{R M_{k+1}}{R M_{k}}-\frac{R M_{k}}{R M_{k-1}}$ exceeds in absolute value the distance between the indicator values $\frac{S_{k}}{S_{k}^{\nabla}}-\frac{S_{k-1}}{S_{k-1}^{\nabla}}$.

Thus the prediction of the peak sunspot number for cycle 24 is made on the basis of the following expression $\frac{R M_{23}}{R M_{22}}-\frac{R M_{24}}{R M_{23}}>\frac{S_{22}}{S_{22}^{V}}-\frac{S_{23}}{S_{23}^{V}}$.

The solution of this inequality indicates that the upper bound for cycle 24 will be 72 .

To reconstruct the values of $R M_{k+1}$ we used earlier the linear relationship between the distances $\frac{R M_{k+1}}{R M_{k}}-\frac{R M_{k}}{R M_{k-1}}$ and $\frac{S_{k}}{S_{k}^{\nabla}}-\frac{S_{k-1}}{S_{k-1}^{\nabla}}$ taking into account the tendency of $\frac{S_{k}}{S_{k}^{\nabla}}-\frac{S_{k-1}}{S_{k-1}^{\nabla}}$ to decrease with increase of $k$ (Podladchikova et al. 2008). However, after the minimum of cycle 23 had fallen, it became clear that this cycle differs much from the previous ones. It is very prolonged and its descending time, equal to 8.62 years, is the largest for all the cycles from 1 to 23 , excepting the cycle 4 , which had the longest descending time, equal to 10.2 years.

Therefore now we make a more conservative prediction for cycle 24 based on the stable regularities of all the cycles irrespective of $k$.

\section{Conclusion and discussion}

Two relevant indicators that capture the intrinsic variability of the declining phase of the sunspot cycles were used for the prediction of the peak value for cycle 24 . The first indicator determines if the amplitude of the next cycle will be larger or smaller compared with the current one and is validated by a perfect agreement for all the cycles from 1 to 23 . It indicates that cycle 24 will be weaker than the previous one.

The second indicator that is less sensitive to the errors of smoothing was used for the quantitative estimation of the 


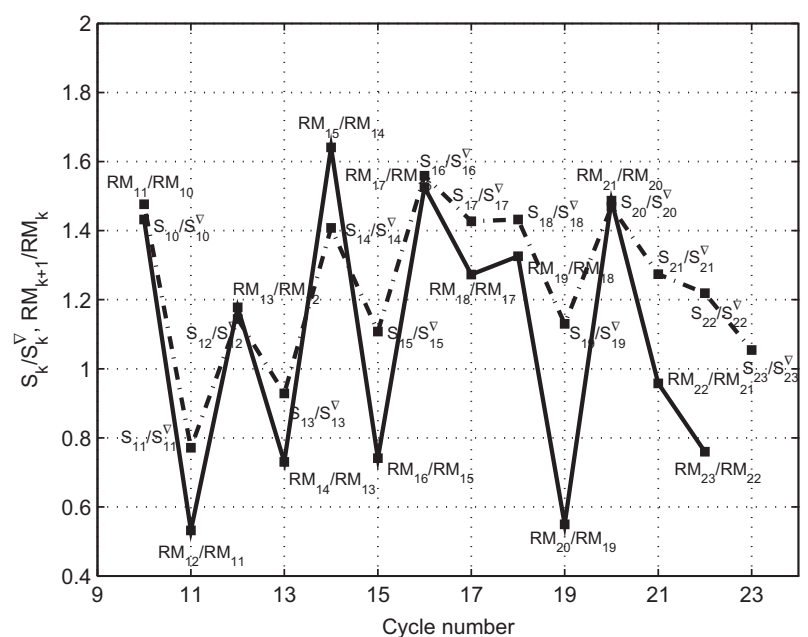

Fig. 3. The indicators $\frac{S_{k}}{S_{k}^{V}}$ (dashed line) and ratio $\frac{R M_{k+1}}{R M_{k}}$ (solid line). The dynamics of $\frac{S_{k}}{S_{k}^{\nabla}}$ substantially resembles those of $\frac{R M_{k+1}}{R M_{k}}$.

maximum sunspot number of cycle 24. According to this second indicator we get an upper bound of the sunspot number for solar cycle 24 and predict that its peak value will not exceed 72 .

Many authors predict that the solar activity for cycle 24 will be lower than cycle 23 (Schatten 2005; Choudhuri et al. 2007; Javaraiah 2007).

Our result is in agreement with the forecast based on the polar field precursor that cycle 24 will be the smallest in the last 100 years (Svalgaard et al. 2005). The longest descending time of cycle 23 with the exception of cycle 4 (that precedes the Dalton minimum) can be evidently considered as the indicator of the exceptionality of cycle 24 . Using a solar dynamo precursor method, Schatten \& Tobiska (2003) predict a rapid decline starting with cycle 24. Maris et al. (2003) observing the flare energy release during the declining phase of the precedent cycle indicated that the Sun might be heading toward a "Maunder" type of minimum.

Acknowledgements. The authors are grateful to the team of the "Solar Influences Data analysis Center" (SIDC) at the Royal Observatory of Belgium (ROB) for data sets and to the referees for helpful comments.

\section{References}

Brajša, R., H. Wöhl, A. Hanslmeier, G. Verbanac, D. Ruždjak, E. Cliver, L. Svalgaard, and M. Roth, A Prediction for the $24^{\text {th }}$ Solar Cycle, Cent. Eur. Astrophys. Bull., 33, 95-98, 2009.
Cameron, R., and M. Schüssler, Solar cycle prediction using precursors and flux transport models, The Astrophys. J., 659 (1), 801-811, 2007.

Cameron, R., and M. Schüssler, A robust correlation between growth rate and amplitude of solar cycles: Consequences for prediction methods, The Astrophys. J., 685, 1291-1296, 2008.

Choudhuri, A.R., P. Chatterjee, and J. Jiang, Predicting solar cycle 24 with a solar dynamo model, Phys. Rev. Lett., 98, 131103, 2007.

Feynman, J., Geomagnetic and solar wind cycles, 1900-1975, J. Geophys. Res., 87, 6153-6162, 1982.

Gonzalez, G., and K.H. Schatten, Using geomagnetic indices to forecast the next sunspot maximum, Solar Phys., 114, 189-192, 1987.

Hathaway, D.H., Solar cycle forecasting, Space Sci. Rev., 144, 401-412, 2009.

Hathaway, D.H., R.M. Wilson, and E.J. Reichmann, A synthesis of solar cycle prediction techniques, J. Geophys. Res., 104 (A10), $22.375-22.388,1999$

Javaraiah, J., North-south asymmetry in solar activity: Predicting the amplitude of the next solar cycle, Mon. Not. R. Astron. Soc., 377, L34-L38, 2007.

Kane, R.P., Prediction of solar cycle maximum using solar cycle lengths, Sol. Phys., 248 (1), 203-209, 2008.

Lantos, P., The skewness of a solar cycle as a precursor of the amplitude of the next, Solar Phys., 236, 199-205, 2006.

Maris, G., M.D. Popescu, and D. Besliu, Solar cycle 23: Forecasts and observations, Rom. Astron. J., 13 (2), 139-142, 2003.

Ohl, A.I., and G.I. Ohl, A new method of very long-term prediction of solar activity, NOAA Solar-Terr. Pred. Proc., 2, 268-263, 1979.

Podladchikova, T., B. Lefebvre, and R. Van der Linden, Integral activity of the declining phase of sunspot cycles as precursor of the next cycle, J. Atm. Solar-Terr. Phys., 70, 277-284, 2008.

Ramaswamy, G., Sunspot cycles and solar activity forecasting, Nature, 265, 713-715, 1977.

Sargent, H.H., A prediction for the next sunspot cycle, $28^{\text {th }}$ IEEE Vehicular Tech Conf. Proc., 490-496, 1978.

Schatten, K. Fair space weather for solar cycle 24, Geophys. Res. Lett., 32, L21106, 2005.

Schatten, K., and S. Sofia, Forecast of an exceptionally large evennumbered solar cycle, Geophys. Res. Lett., 14, 632-635, 1987.

Schatten, K.H. and W.K. Tobiska, Solar activity heading for a Maunder minimum?, Bull. Am. Astro. Soc., 35, 817, 2003.

Schatten, K.H., P.H. Scherrer, L. Svalgaard, and J.M. Wilcox, Using dynamo theory to predict the sunspot number during solar cycle 21, Geophys. Res. Lett., 5, 411-414, 1978.

Svalgaard, L., E.W. Cliver, and Y. Kamide, Cycle 24: the smallest sunspot cycle in 100 years?, Geophys. Res. Lett., 32, 1, L01104, 2005.

Thomson, R.J., A technique for predicting the amplitude of the solar cycle, Solar phys., 148, 383-388, 1993.

Wilson, R.M., D.H. Hathaway, and E.J. Reichmann, An estimate for the size of cycle 23 based on near minimum conditions. J. Geophys. Res., 103, 6595-6603, 1998. 